\title{
CARACTERIZAÇÃO E ESTUDO DE ALTERABILIDADE DO BUSTO DE JESUS CRISTO
} PERTENCENTE AO MOSTEIRO DE SÃO BENTO, RIO DE JANEIRO

\author{
Luanna C. Rebecchi de Moura ${ }^{1}$, Carla Felix ${ }^{2}$, Roberto Carlos da Conceição Ribeiro ${ }^{3}$, \\ Daniele Pereira Dalto ${ }^{4}$, Kátia Leite Mansur ${ }^{5}$ \\ 1 - Geóloga, Bolsista PCI do CETEM; 2 - Estudante de Geologia, UFRJ; 3 - Engenheiro Químico, D.Sc, Pesquisador do CETEM; \\ 4 - Engenheira Química, M.Sc, Bolsista PCI CETEM; 5 - Geóloga, professora da UFRJ \\ Icmoura@cetem.gov.br
}

Resumo: O conjunto edificado formado pela Igreja de Nossa Senhora de Monserrat e Mosteiro de São Bento foi o principal empreendimento da cidade do Rio de Janeiro no século XVII, sendo um importante representante deste período e uma das poucas construções barrocas dentro do panorama luso-brasileiro cuja autenticidade foi preservada até os dias atuais. Nos jardins do claustro, próximo a um pequeno lago, existe um busto de Jesus Cristo talhado em rocha e uma base quadrada que suporta o busto de aspecto semelhante. Em vistas das poucas informações disponíveis sobre estas peças foram realizados ensaios não destrutivos para a caracterização delas e de seus danos, além da lavagem e coleta de água para avaliação do pH, análise de sais e de sujidade das partes separadamente. No busto foram realizadas 35 medições de dureza (resultados entre 333 e 574 HLD), 97 de cor e brilho, 56 de fluorescência de raios-X portátil (FRX) e 9leituras de velocidade ultrassônica (variando de 1.230 a $7.139 \mathrm{~m} / \mathrm{s}$ ). Para a base foram avaliados 28 pontos de dureza (valores entre 110e 309 HLD), 67 de cor e brilho, 43 de fluorescência de raios-X portátil. Devido ao histórico incompleto das peças, optou-se também pelas análises de difração e FRX laboratoriais. Visualmente foi possível constatar que a parte superior do busto apresenta crosta negra em diferentes pontos e a peça de base apresentou perda de massa formando pequenos veios. A coleta de material ocorreu na base do busto e na parte interna da base quadrada de forma a preservar a peça. Os resultados apontam que as peças foram esculpidas em mármore calcítico, e a água de lavagem continha sulfetos, sulfatos e nitratos, além de íons de cálcio, tais poluentes justificam a presença de gipsita no lugar de calcita na base quadrada, o que condiz com o aspecto visual mais danificado da mesma (pequenos veios formados).

Palavras Chave: rochas ornamentais, alterabilidade, Busto de Jesus Cristo do Mosteiro de São Bento

Abstract: CHARACTERIZATION AND STUDY OF ALTERABILITY OF THE BUST OF JESUS CRISTO BELONGING TO THE CONVENT OF SÃO BENTO, RIO DE JANEIRO. The group of buildings formed by the Church of Our Lady of Monserrat and Monastery St. Benedict was the main project of the city of Rio de Janeiro in the seventeenth century, and an important representative of this period and one of the few Baroque buildings within the Luso-Brazilian panorama whose authenticity has been preserved until the present day. In the cloister gardens, next to a small lake there is a bust of Jesus Christ carved in rock and a square base that supports the similar-looking bust. In view of the limited information available on these pieces were performed non-destructive testing to characterize them and their damage, as well as washing and collecting this water for evaluation of $\mathrm{pH}$, salts analysis and dirt from parts separately. In the bust were performed 35 hardness measurements (results between 333 and 574 HLD), 97 color and gloss, 56 fluorescence X-ray laptop (XRF) and 9 readings ultrasonic velocity (range $1230-7139 \mathrm{~m} / \mathrm{s}$ ) . For the base were measured 28 points of hardness values (between 110 and 309 HLD) 67 of color and brightness, fluorescence $43 \mathrm{X}$-ray laptop. Due to incomplete history of parts, it was chosen also by diffraction analyzes and laboratory XRF. Visually it was found that the upper part of the bust displays black crust at various points and the base part showed mass loss forming small veins. The collection of material occurred in the bust base and inside the square base to preserve the piece. The results show that the pieces were carved in calcite marble, and the wash water contained sulfides, sulfates and nitrates, and calcium ions, such pollutants justify the presence of gypsum in place of calcite on square base, which is consistent with the more damaged visual appearance (small veins formed)

Keywords: dimension stone, alterability, Monastery of São Bento

\section{INTRODUÇÃO}

A peça foco do presente trabalho é um Busto de Jesus Cristo esculpido em rocha de coloração branca, localizado no jardim interno na área do claustro dos monges dentro do Mosteiro de São Bento. A peça foi doada ao Mosteiro, mas não há registro histórico. Na lateral da peça está assinado o nome "Ir. Pattrich" e o ano de 1893.

O Mosteiro de São Bento está localizado na região Portuária da cidade do Rio de Janeiro, próximo à Praça Mauá. O Busto encontra-se numa parte com acesso restrito, num pequeno bosque no claustro dos monges, conforme Figura 1.

\section{OBJETIVO}

O presente trabalho tem como objetivo a caracterização tecnológica do Busto de Jesus Cristo e de sua base quadrada pertencente ao Mosteiro de São Bento.

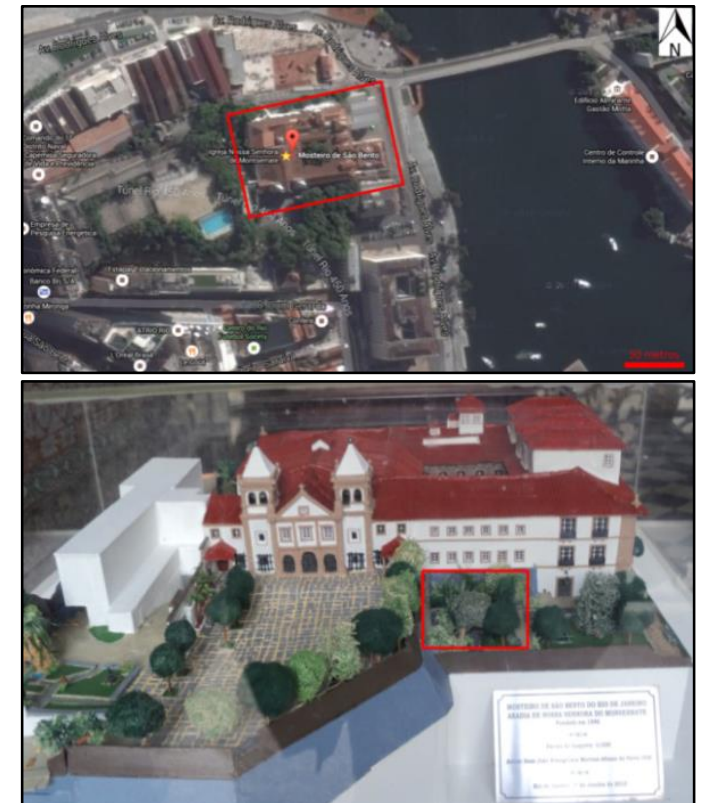

Figura 1. Localização do Mosteiro de São Bento, localização do bosque (fonte: Google Maps). 


\section{MATERIAIS E MÉTODOS}

Para fins de melhor avaliação, o Busto de Cristo foi separado da base quadrada e os ensaios e interpretação dos resultados foram realizados separadamente. Nas Figuras 2 e 3 estão indicados os pontos de análise. É importante destacar que a base quadrada é um quadrado vazado e não é responsável por sustentar a estátua.

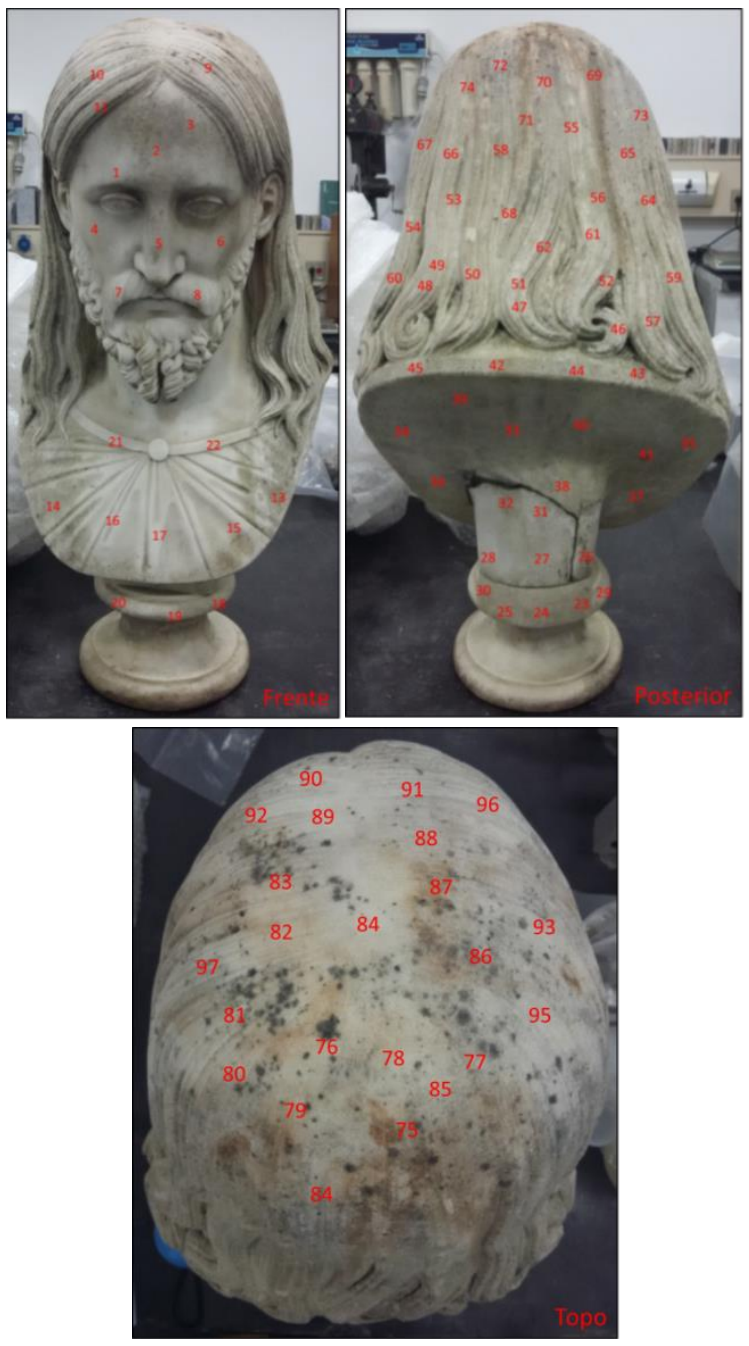

Figura 2. Indicação dos pontos onde foram realizados os ensaios.

Para os ensaios de dureza e velocidade ultrassônica foram utilizados dados obtidos em amostras de mármores sãos que se encontravam no laboratório, identificados por M-01, M-02 e M-03.

\subsection{Caracterizações química (FRX) e mineralógica (DRX)}

Devido ao histórico incompleto das peças, optouse pela raspagem de quantidade bem pequena para a realização das técnicas de fluorescência de raios- $X$ e difração de raios-X (DRX)no laboratório de análises químicas do CETEM. A primeira realizada para determinação dos elementos químicos presentes nas amostras e a segunda para determinação dos compostos mineralógicos. Para determinação de teores de enxofre foi utilizado o analisador elementar de carbono e enxofre LECO SC632.

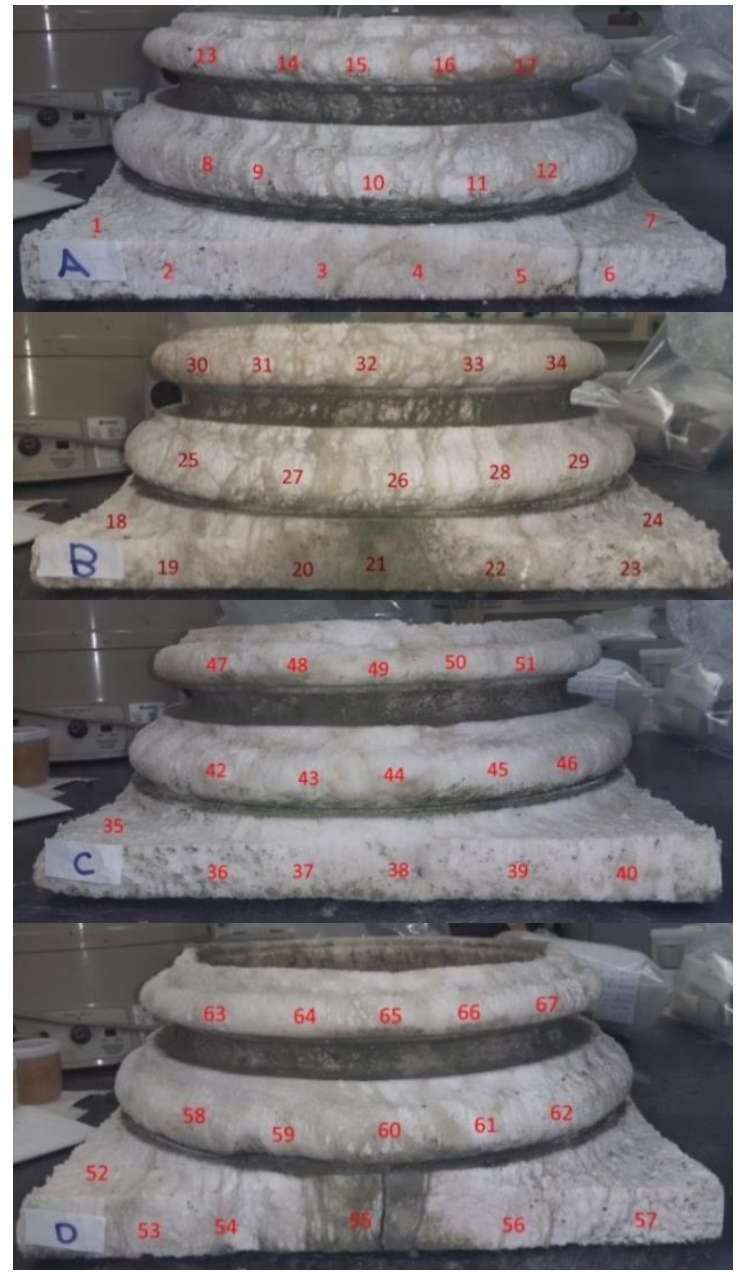

Figura 3. Indicação dos pontos onde foram realizados os testes de dureza, cor e brilho e FRX portátil na base quadrada.

\subsection{Análise de sais e sujidades}

O busto e a base foram lavados com água deionizada e a sujidade superficial foi removida por meio de uma escova de cerdas macias. As águas provenientes dessa lavagem foram armazenadas em potes de polipropileno estéril para averiguação dos íons de interesse por meio de espectrometria de emissão ótica com plasma induzido (ICP-OES). Ressalta-se que as água obtidas com a lavagem do Busto não foram misturadas com as obtidas na lavagem da Base. Essas águas foram analisadas também quanto ao $\mathrm{pH}$ para determinação da acidez/alcalinidade das peças utilizando-se 0 pHmetro digital da marca Thermo Scientific, modelo Orion 4-Star.

\subsection{Determinação de dureza}

Para medição da dureza, os testes foram realizados com um Durômetro Proceq, modelo Equotip3. A dureza foi medida em 84 pontos do Busto e 57 pontos da base quadrada, seguindo a numeração apresentada anteriormente. Os 
resultados foram comparados com os obtidos em ensaios em mármores sãos.

\subsection{Ensaio de absorção com tubo de Karsten}

Mais conhecido por "Método do Cachimbo", este ensaio mede a quantidade de água absorvida por uma superfície de alvenaria ou revestimento, durante um período de tempo específico. No Brasil este ensaio não é normalizado, mas vem sendo utilizado para determinar a impermeabilidade de superfícies verticais.

O cachimbo de plástico consiste em um dispositivo graduado a cada 0,1 $\mathrm{mm}$ em formato de "L", com uma borda plana e circular no fundo com $2,5 \mathrm{~cm}$ de diâmetro totalizando aproximadamente $5,0 \mathrm{~cm}^{2}$ de área de contato, a qual é fixada à superfície do revestimento com massa de adesão. Para o inicio do ensaio é necessário preencher o "cachimbo" com água até o nível de referência zero, o tempo vai sendo marcado e a diminuição na altura da água ao longo do tempo é interpretada como indicação da vulnerabilidade do material à penetração da água.

\subsection{Determinação de velocidade ultrassônica}

A determinação da velocidade de propagação de ondas ultrassônicas permite avaliar, indiretamente, o grau de alteração e coesão das rochas (Torquato \& et. al., 2002). Esse procedimento foi baseado na norma ABNT NBR 8802/13 utilizando-se o equipamento PUNDIT (Portable Ultrassonic Non Destructive Digital Indicating Tester) da fabricante Proceq, modelo PL-200.Foram escolhidas regiões com superfícies planas para possibilitar o encaixe dos transdutores e o arranjo de transmissão direta. A superfície muito irregular da base quadrada não permitiu a realização deste ensaio.

\section{RESULTADOS E DISCUSSÕES}

\subsection{Caracterizações química (FRX) e} mineralógica (DRX)

Os dados obtidos pela análise de fluorescência de raios- $X$ demonstram que o busto é composto por $49,90 \%$ de óxido de cálcio e possui perda ao fogo de $44,30 \%$. Por meio do difratograma (Figura 4A) é possível concluir que se trata de um mármore calcítico. Já a base quadrada apresenta teor de $30,90 \%$ de óxido de cálcio, $22,00 \%$ de perda ao fogo e um teor de $22,00 \%$ de enxofre. No difratograma da Figura 4B é possível observar que a base quadrada apresenta predominantemente picos de gipsita em maior intensidade do que os picos de carbonato de cálcio, o que acaba por justificar uma quantidade tão alta de enxofre detectado durante a caracterização química.

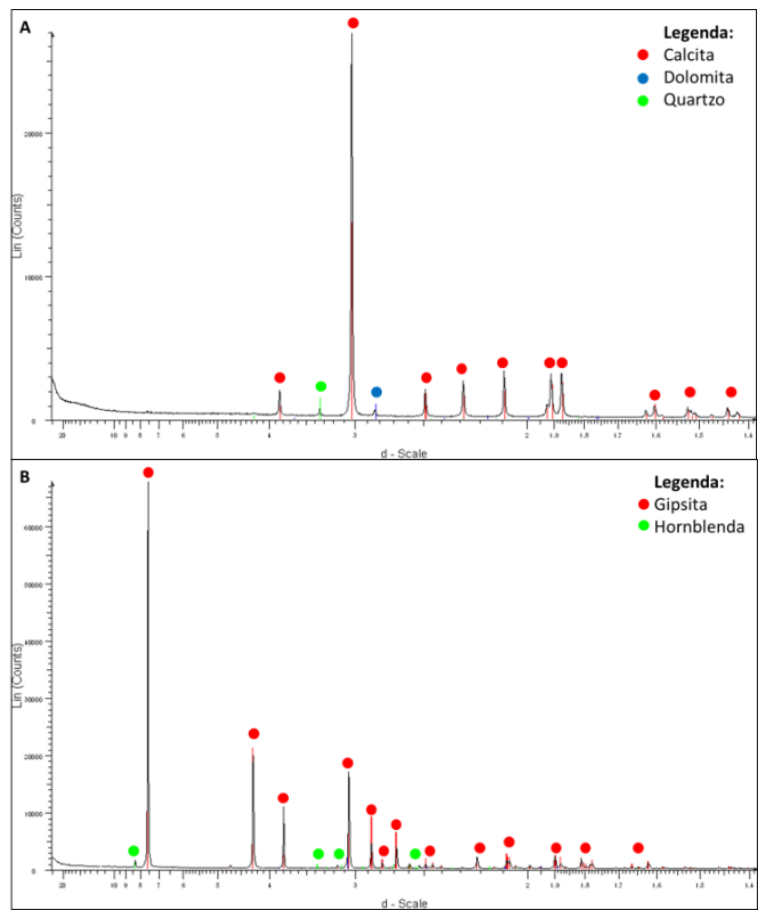

Figura 4. A-Difratograma do busto; B-Difratograma da base quadrada.

\subsection{Análise de sais e sujidades}

$\mathrm{Na}$ Tabela 1 estão apresentados os resultados da análise química da água de lavagem. Observam-se altos teores de cálcio e sulfeto, possivelmente relacionados à alteração das rochas, gerando a gipsita encontrada na análise de FRX. Observam-se elevados teores de nitrato, chegando a cerca de 40 mg.L-1, possivelmente devido ao efeito de fezes de animais.

Tabela 1. Análise química (mg.L-1) dos íons encontrados na água de lavagem.

\begin{tabular}{c|c|c}
\hline Íons & Base Quadrada & Busto \\
\hline $\mathbf{N a}^{+}$ & 3,60 & 8,60 \\
\hline $\mathbf{A l}^{3+}$ & $<0,007$ & $<0,007$ \\
\hline $\mathbf{C a}^{2+}$ & 369,00 & 33,40 \\
\hline $\mathbf{C l}^{-}$ & 6,00 & 13,00 \\
\hline $\mathbf{K}^{+}$ & 0,41 & 11,00 \\
\hline $\mathbf{M g}^{+2}$ & 0,05 & 1,20 \\
\hline $\mathbf{S 2}^{-}$ & 300,00 & 150,00 \\
\hline $\mathbf{( S O 4 )}^{\mathbf{2}}$ & 1,00 & 40,00 \\
\hline (NO3) $_{\mathbf{1}}^{-}$ & 17,00 & 39,00 \\
\hline
\end{tabular}

As quantidades de sulfeto e sulfato encontrados são indicativas da poluição atmosférica a qual o Mosteiro está sujeito. Esta poluição pode ter sido agravada pelos gases emitidos pelos veículos que trafegavam na Avenida Perimetral, hoje extinta.

$\mathrm{Em}$ relação ao $\mathrm{pH}$ para a água de lavagem do busto foi de 7,38 e para a base quadrada foi de 5,55. $\mathrm{O}$ valor ácido encontrado para a base pode estar 
relacionado aos maiores valores de sulfetos determinados nesta peça. Já no busto, o valor alcalino pode estar relacionado com o excesso de cálcio desagregado da rocha.

\subsection{Determinação de dureza}

Na Tabela 2 estão apresentados os resultados relativos ao ensaio do busto, além dos obtidos para os padrões (M-01, 02 e 03). Verifica-se que os valores encontrados na base são menores que os valores do busto. O menor valor encontrado foi de $110 \mathrm{HDL}$, na base, enquanto que o valor $574 \mathrm{HDL}$ foi encontrado no busto. As diferenças relativas nos valores podem indicar que a base está sendo mais afetada pelos agentes de intemperismo do que o busto. Na Tabela 3 estão apresentados os resultados obtidos para a base quadrada.

A média na região da frente do busto ficou em $489,07 \mathrm{HDL}$, na parte posterior $452,19 \mathrm{HDL}$ e no topo do busto 411,20 HDL, sendo que essa área é visivelmente a mais afetada, apresenta manchas e pequenos buracos, que podem decorrer de dissoluções da rocha.

Tabela 2. Valores de dureza do busto.

\begin{tabular}{|c|c|c|c|c|c|}
\hline Região & Ponto & Medições (hdl) & Região & Ponto & Medições (hdl) \\
\hline \multirow{14}{*}{ Frente } & 2 & 481 & \multirow{16}{*}{ Posterior } & 24 & 529 \\
\hline & 4 & 571 & & 26 & 429 \\
\hline & 5 & 536 & & 27 & 497 \\
\hline & 6 & 520 & & 33 & 503 \\
\hline & 7 & 512 & & 34 & 501 \\
\hline & 9 & 449 & & 35 & 462 \\
\hline & 10 & 333 & & 36 & 476 \\
\hline & 13 & 472 & & 37 & 477 \\
\hline & 14 & 428 & & 38 & 497 \\
\hline & 15 & 543 & & 39 & 427 \\
\hline & 16 & 494 & & 40 & 478 \\
\hline & 19 & 411 & & 42 & 457 \\
\hline & 21 & 523 & & 48 & 361 \\
\hline & 22 & 574 & & 59 & 348 \\
\hline \multirow{5}{*}{ Topo } & 80 & 423 & & 65 & 342 \\
\hline & 83 & 464 & & 71 & 451 \\
\hline & 84 & 404 & \multirow{3}{*}{$\begin{array}{l}\text { Valores médios } \\
\text { padrões } \\
\text { (materiais sãos) }\end{array}$} & M-01 & 477 \\
\hline & 85 & 364 & & $\mathrm{M}-02$ & 481 \\
\hline & 88 & 401 & & M-03 & 600 \\
\hline
\end{tabular}

A média do lado $A$ da base ficou em 188,14, lado B em 172,28 HDL, lado C em 164,57 e lado D 201,71 HDL.

Tabela 3. Valores de dureza da base quadrada.

\begin{tabular}{|c|c|c|c|c|c|}
\hline Lado & Ponto & $\begin{array}{l}\text { Medições } \\
\text { (HDL) }\end{array}$ & Lado & Ponto & $\begin{array}{c}\text { Medições } \\
\text { (HDL) }\end{array}$ \\
\hline \multirow{7}{*}{$A$} & 2 & 207 & \multirow{7}{*}{ B } & 19 & 204 \\
\hline & 4 & 309 & & 21 & 165 \\
\hline & 6 & 198 & & 23 & 150 \\
\hline & 8 & 168 & & 27 & 169 \\
\hline & 10 & 186 & & 28 & 158 \\
\hline & 14 & 132 & & 31 & 174 \\
\hline & 17 & 117 & & 33 & 186 \\
\hline \multirow{7}{*}{$C$} & 36 & 138 & \multirow{7}{*}{ D } & 53 & 169 \\
\hline & 38 & 173 & & 55 & 220 \\
\hline & 40 & 204 & & 57 & 224 \\
\hline & 43 & 110 & & 59 & 179 \\
\hline & 45 & 156 & & 61 & 197 \\
\hline & 48 & 172 & & 64 & 234 \\
\hline & 51 & 199 & & 66 & 189 \\
\hline
\end{tabular}

Quando os valores dos lados da base quadrada são comparados aos obtidos no busto e nos padrões de laboratório, pode-se notar que os valores da base são substancialmente menores (média de 181,67 HDL).A aparência desta parte da escultura é mais irregular que o busto, sendo marcada por veios de aspecto vítreo e relevo. Estes relevos podem dificultar o ensaio e promover a obtenção de valores mais baixos.

O busto por outro lado apresenta valores relativamente bons quando comparados aos padrões, com exceção de alguns pontos onde as durezas ficaram inferiores a $400 \mathrm{HDL}$.

\subsection{Ensaio de absorção com tubo de Karsten}

Na base quadrada o ensaio foi realizado no lado $B$ entre os pontos 20 e 21 e também sob a base, próximo ao lado $B$. Já no busto o ensaio foi realizado na base, no topo (ponto 89), na parte da frente na região do ponto 16 e na testa (ponto 2 ).

Em relação ao busto, os primeiros ensaios foram em sua base e em seu topo, uma vez que a estátua encontrava-se apoiada horizontalmente sobre uma espuma grossa que a protegia do contato com o piso. Este ensaio foi feito por uma hora e, devido à ausência de resultado, foi prolongado por outra hora. Embora ao final de duas horas não tenha ocorrido observação de decréscimo da coluna de água o ensaio foi finalizado, os aparatos foram removidos, a estátua foi colocada em posição vertical e foram selecionados os pontos da testa e peito para novo ensaio. Novamente após uma hora de ensaio, nenhum decréscimo foi notado e optouse pela extensão do tempo de ensaio, acidentalmente o tubo de Karsten foi removido do ponto 16, e dessa forma o ensaio foi estendido apenas para o tubo localizado no ponto 2 . Com um tempo total de ensaio de três horas e nenhum movimento da coluna d'água o ensaio foi finalizado.

Exatamente o mesmo resultado foi obtido para os pontos ensaiados na base quadrada, duas horas após a colocação do tubo de Karsten no Lado B, região 20/21, nenhum movimento da coluna de água foi percebido e o ensaio foi finalizado. Houve a troca na posição do tubo, passando para a parte inferior da base quadrada e após 3 horas de ensaio, sem movimentação da coluna de água, o ensaio foi finalizado.

Em laboratório foi realizado este mesmo ensaio nos mármores $\mathrm{M}-01, \mathrm{M}-02$ e $\mathrm{M}-03$. Após uma hora de ensaio não foram observados decréscimos na coluna de água em nenhuma das amostras, o ensaio foi encerrado.

Da ausência de decréscimo da coluna de água nos tubos duas hipóteses podem ser concebidas, a primeira de que a peça está tão saturada que não há 
absorção de água e a segunda de que o mármore não tem absorção expressiva neste tipo de ensaio.

\subsection{Determinação de velocidade ultrassônica}

Segundo a classificação de danos de Köhler, 1991 (in Ruedrichet al, 2013), mármores de Carrara frescos possuem velocidade superior a $5.000 \mathrm{~m} / \mathrm{s}$, estas informações são coincidentes com as de Sarpünet.al.(2009) para mármores com porosidade inferior a $0,5 \%$ e cerca de $50 \%$ de carbonato de cálcio.

Tabela 4. Velocidade de Pulso Ultrassônico.

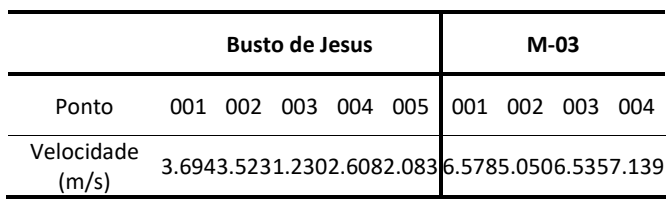

Dessa forma, a comparação dos valores da literatura com os obtidos no ensaio para o busto sugere que este mármore não está em seu melhor estado de conservação, todavia não está completamente deteriorado.

\section{CONCLUSÃO}

O busto e a base quadrada são constituídos por um mármore calcítico, embora a base tenha apresentado picos de maior intensidade relativos a gipsita. Foram observadas concentrações de sulfetos e sulfatos, possivelmente provenientes da poluição atmosférica e a presença de cálcio, que pode ser relacionada a dissoluções sucessivas, principalmente na região da base, que inclusive é visualmente evidente. Esse aspecto degradado da base somado ao resultado químico que indica a presença de gipsita pode ser responsável pela discrepância observada nos dados obtidos na avaliação da dureza superficial, na qual o Busto possui média de 461,1 HLD enquanto que a base quadrada tem valores em torno de 181,7 HLD, possivelmente as superfícies irregulares da base influenciaram negativamente neste ensaio, que depende de superfícies planas. A não percepção de decréscimo da coluna de água precisa ser estudada a fundo. Por fim, os valores constatados para as velocidades ultrassônicas sugerem um estado de alteração não tão avançado quanto o esperado durante o estudo, já que as peças encontravam-se em um jardim com densa vegetação e sem qualquer tipo de proteção adicional contra as intempéries e a poluição atmosférica da cidade, em especial, da extinta Avenida Perimetral cujo intenso trafego de automóveis teve fim a menos de dois anos.

\section{AGRADECIMENTOS}

Ao CETEM pela infraestrutura, ao CNPq pelo apoio financeiro, ao Mosteiro de São Bento e a Empresa Construtora Terreng pela colaboração e a todos que contribuíram de forma direta ou indireta para a realização deste trabalho, especial agradecimento a técnica em química Evelyn Xavier.

\section{REFERÊNCIAS BIBLIOGRÁFICAS}

ABNT. ASSOCIAÇÃO BRASILEIRA DE NORMAS TÉCNICAS: NBR 8802: Concreto endurecido - Determinação da velocidade de propagação de onda ultrassônica, Rio de Janeiro.2013.

Torquato, J. R., \& et. al. (2002): Uso do Pundit na Determinação de Anomalias ultrassônicas em blocos Rochosos. $3^{\circ}$ Simpósio de Rochas Ornamentais do Nordeste, (pp. 20-23). Recife.

Frazão, E. B., \& Farjallat, J. E. (1996). Proposta de Especificação para Rochas Silicáticas de Revestimento. $8^{\circ}$ Congresso Brasileiro de Geologia de Engenharia (pp. 369-380). Rio de Janeiro: ABGE.

Köhler, 1991 (inRuedrichet al, 2013)

Sarpün, I. H.; Özkan, V.; Tuncel, S. (2009): Ultrasonic Determination of Elastic Modulus ofMarbles Relation with Porosity And $\mathrm{CaO} \%$. The 10th International Conference of the Slovenian Society for Non-Destructive Testing: "Application of Contemporary Non-Destructive Testing in Engineering". September1-3, Ljubljana, Slovenia, 119-125. Disponível em: < http://www.ndt.net/article/ndtslovenia2009/PDF/P12.pdf>.

Ruedrich, J.; Knell C.; Enseleit, J.;Rieffel, Y.;Siegesmund,S. (2013): Stability assessment of marble statuaries of the Schlossbrücke(Berlin, Germany) based on rock strength measurementsand ultrasonic wave velocities. Environ Earth Sci 69:1451-1469, DOI 10.1007/s12665-013-2246-x.

Contribuição ao

1‥ Simpósio Brasileiro de Caracterização e Conservação da Pedra 14 a 16 de dezembro de 2016, Congonhas - MG

Nota:

É de responsabilidade da comissão editorial do Simpósio a revisão gramatical, ortográfica, de citações e referências bibliográficas. As normas de submissão podem se diferenciar das desta revista. 